\title{
Spectral Detector CT-Derived Pulmonary Perfusion Maps And Pulmonary Parenchyma Characteristics For The Semiautomated Classification of Pulmonary Hypertension
}

\section{Roman Johannes Gertz ( $\nabla$ roman.j.gertz@gmail.com )}

University of Cologne, Faculty of Medicine and University Hospital Cologne, Department of Radiology Felix Gerhardt

University of Cologne, Faculty of Medicine and University Hospital Cologne, Department of Cardiology Jan Robert Kröger

Johannes Wesling University Hospital, Ruhr University Bochum, Department of Radiology,

Neuroradiology and Nuclear Medicine,

\section{Rahil Shahzad}

Clinical Applications Research, Philips GmbH Innovative Technologies, Aachen

\section{Liliana Caldeira}

Clinical Applications Research, Philips GmbH Innovative Technologies, Aachen

\section{Jonathan Kottlors}

University of Cologne, Faculty of Medicine and University Hospital Cologne, Department of Radiology

Nils Große Hokamp

University of Cologne, Faculty of Medicine and University Hospital Cologne, Department of Radiology

David Maintz

University of Cologne, Faculty of Medicine and University Hospital Cologne, Department of Radiology

\section{Stephan Rosenkranz}

University of Cologne, Faculty of Medicine and University Hospital Cologne, Department of Cardiology Alexander Christian Bunck

University of Cologne, Faculty of Medicine and University Hospital Cologne, Department of Radiology

\section{Research Article}

Keywords: pulmonary, malperfused, thromboembolic, homogenous

Posted Date: September 23rd, 2021

DOl: https://doi.org/10.21203/rs.3.rs-900725/v1 
License: (c) (i) This work is licensed under a Creative Commons Attribution 4.0 International License. Read Full License

Version of Record: A version of this preprint was published at Frontiers in Cardiovascular Medicine on February 28th, 2022. See the published version at https://doi.org/10.3389/fcvm.2022.835732. 


\section{Abstract}

Objectives: To evaluate the usefulness of spectral detector CT (SDCT)-derived pulmonary perfusion maps and pulmonary parenchyma characteristics for the semiautomated classification of pulmonary hypertension $(\mathrm{PH})$.

Methods: A total of 162 consecutive patients with right heart catheter (RHC)-proven $\mathrm{PH}$ of different etiologies as defined by the Nice classification who underwent CT pulmonary angiography (CTPA) on SDCT and 20 patients with an invasive rule-out of $\mathrm{PH}$ were included in this retrospective study. Semiautomatic lung segmentation into normal and malperfused areas based on iodine content as well as automatic, virtual noncontrast-based emphysema quantification were performed. Corresponding

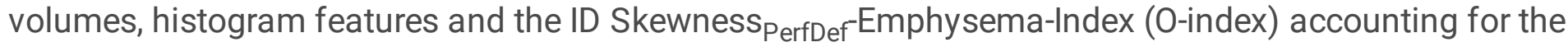
ratio of ID distribution in malperfused lung areas and the proportion of emphysematous lung parenchyma were computed and compared between groups.

Results: Patients with PH showed a significantly greater extent of malperfused lung areas as well as stronger and more homogenous perfusion defects. In Nice class 3 and 4 patients, ID skewness revealed a significantly more homogenous ID distribution in perfusion defects than in all other subgroups. The bindex allowed for further subclassification of subgroups 3 and $4(p<0.001)$, identifying patients with chronic thromboembolic PH (CTEPH, subgroup 4) with high accuracy (AUC: 0.92, 95\%-Cl, 0.85-0.99).

Conclusion: Abnormal pulmonary perfusion in $\mathrm{PH}$ can be detected and quantified by semiautomated SDCT-based pulmonary perfusion maps. ID skewness in malperfused lung areas, and the j-index allow for a classification of PH subgroups, identifying Nice class 3 and 4 patients with high accuracy, independent of reader expertise.

\section{Introduction}

Pulmonary hypertension $(\mathrm{PH})$ describes a rare group of diseases that are defined by an increase in mean pulmonary artery pressure (mPAP) $\geq 25 \mathrm{mmHg}$ at rest as assessed by right heart catheterization (RHC).1]. According to the current guidelines, $\mathrm{PH}$ is classified based on either hemodynamic characteristics (pre-/postcapillary or a combination of both) or clinical presentation. The clinical classification defines five subgroups: pulmonary arterial hypertension (PAH, Nice group 1), PH due to left heart disease (Nice group 2), PH due to lung disease and/or hypoxia (Nice group 3), chronic thromboembolic PH (CTEPH, Nice group 4) and PH with unclear/multifactorial mechanisms (Nice group $5)$.

As a result of the broad variety of underlying pathomechanisms and the overlapping presentation in $\mathrm{RHC}$ (elevated precapillary pressure in subgroups 1, 3, 4 and 5), a repertory of diagnostic tests (e.g., echocardiography, RHC, pulmonary function testing and blood gases, V/Q-scintigraphy) is recommended to achieve the final diagnosis in patients with suspected PH.1]. Timely diagnosis is of crucial importance, 
as it not only defines therapy but also $\mathrm{PH}$, regardless of the cause, is associated with poor prognosis.25].

With the introduction of dual energy CT (DECT), mapping of pulmonary perfusion based on the different absorption characteristics of iodine and lung parenchyma has become available.6]. The generated iodine density images (IDIs) are considered a sufficient surrogate parameter to estimate organ perfusion[7, 8] and have proven to provide information on pulmonary perfusion in the setting of acute pulmonary embolism as well as in CTEPH with comparable or even superior accuracy to V/Q-scintigraphy.6, 9-16]. In comparison to V/Q-scintigraphy, which remains the cornerstone to screen for CTEPH, DECT offers the advantage of allowing a comprehensive analysis of the lung parenchyma, pulmonary perfusion and vessel anatomy in a single examination.11, 14, 15, 17]. With regard to the complete spectrum of $\mathrm{PH}$, current data suggest that V/Q-scintigraphy 18] and DECT-based pulmonary perfusion maps might aid in the differentiation of $\mathrm{PH}$ subgroups, as different types of perfusion abnormalities correlate with $\mathrm{PH}$ etiology.19-21]. In addition to the evaluation of pulmonary perfusion and vasculature in a "one-stopexamination", DECT offers the unique possibility of quantifying parenchymal lung disease, a potential cause of $\mathrm{PH}$, based on virtual noncontrast (VNC) images without the necessity of extra radiation exposure. Various studies indicate a comparable accuracy of VNC-based emphysema quantification compared to real noncontrast images, 22, 23], which have also been shown to discriminate PH due to lung disease and/or hypoxia from PAH.24].

Notwithstanding these promising results, according to the current guidelines, (DE)CT only plays a supportive role in the diagnostic work-up of PH.1]. This appears reasonable, as its diagnostic accuracy excessively depends on reader expertise.21]. In addition, current data are either limited due to the small sample size and/or rely on time-consuming manual image interpretation, inheriting the limitation of intraand interreader variability.14, 15, 21].

A recently developed software application for volumetric iodine quantification enables iodine quantification per voxel for a 3D dataset acquired on a dual-layer CT platform (spectral detector CT, SDCT).25]. This allows for a semiautomatic, threshold-based segmentation of the lung into normal and malperfused areas based on iodine concentration. Given the potential diagnostic merit of pulmonary perfusion in $\mathrm{PH}$, the aim of our study was to evaluate whether semiautomatically derived volumetric parameters of SDCT-based pulmonary perfusion maps can aid the diagnosis and classification of $\mathrm{PH}$.

\section{Methods}

\subsection{Study population}

The study cohort consisted of 201 consecutive patients who had been referred to the Department of Cardiology of the University Hospital of Cologne for PH screening between May 2016 and May 2019 and underwent CT pulmonary angiography (CTPA) on SDCT. In accordance with the $2015 \mathrm{ESC} / \mathrm{ERS}$ guidelines, patients with suspected $\mathrm{PH}$ underwent $\mathrm{RHC}$, ventilation/perfusion scintigraphy (V/Q scan) and 
further testing.1]. The final diagnosis was reached by expert consensus based on all available diagnostic tests, including CTPA. Twenty of these 201 patients with an invasive rule-out of $\mathrm{PH}$ via RHC (mPAP $<25$ $\mathrm{mmHg}$ ) served as a control cohort.

This retrospective study was approved by the local institutional review board (Ethics committee of the Faculty of Medicine from the University of Cologne, Cologne, Germany). Due to the retrospective design of the study the local institutional review board waived necessity for informed consent. All clinical investigations were conducted in accordance with the Declaration of Helsinki.

\subsection{Image acquisition and reconstruction}

CT data were acquired on a clinically available spectral detector CT (IQon, Philips Healthcare). All patients received an intravenous $50 \mathrm{ml}$ bolus of contrast media (300 mg iodine/ml, Accupaque, GE Healthcare) followed by a $40 \mathrm{ml} \mathrm{NaCl}$ flush injected with a flow rate of $4 \mathrm{ml} / \mathrm{s}$. Scanning was initiated with a delay of $4.9 \mathrm{~s}$ after an attenuation of $150 \mathrm{HU}$ was reached in the MPA. The acquisition parameters were as follows: slice collimation $64 \times 0.625 \mathrm{~mm}$; rotation time $0.33 \mathrm{~s}$; tube potential $120 \mathrm{kV}$, automatic tube current modulation was used, reference mAs 75. For all reconstructions, a soft tissue kernel (B, Philips Healthcare) and a dedicated spectral reconstruction algorithm were used (Spectral, Philips Healthcare). Images were reconstructed in axial orientation with a slice thickness of $1 \mathrm{~mm}$ and a slice overlap of 0.5 $\mathrm{mm}$. Matrix was set to $512 \times 512$. In addition to conventional images, which are identical to images reconstructed with the vendors hybrid-iterative reconstruction algorithm [26] (iDose4, Philips Healthcare), iodine maps and virtual noncontrast images were reconstructed.

\subsection{Image analysis}

\subsubsection{SDCT-based pulmonary perfusion}

Automatic segmentation of the lung was performed using the commercially available software solution (Intellispace Portal, COPD tool, Version 10, Philips Healthcare). In case of insufficient segmentation, lung volumes were manually edited. Subsequently, lung volumes were semiautomatically segmented into normal and malperfused areas via threshold segmentation based on the IDIs using dedicated software for volumetric iodine quantification (ISD, ThresholdSegmentation (1.1) Philips Intellispace Release 11). Three lung areas were defined as follows: malperfused: voxels with an iodine density (ID) of less than $5 \%$ of the MPA, normal perfused: voxels with an ID of more than $5 \%$ of the MPA and less than $50 \%$ of the left atrium (LA), vessel compartment: voxels with an ID of more than $50 \%$ of the LA. Mean IDs in the MPA and LA were measured by manually drawing a ROI that accounted for at least $50 \%$ of the vascular/atrial area at the largest diameter. The following parameters were computed from histogram analysis for normal and malperfused lung areas: mean ID, ID kurtosis and ID skewness. Values for mean ID are given as standardized values calculated by division by the mean ID of the feeding vessel (MPA), as described previously.27]. Figure 1 illustrates the effect of ID distribution on ID skewness.

\subsubsection{Lung emphysema quantification}


Lung emphysema was quantified based on VNC images using a commercially available software solution (Intellispace Portal, COPD tool, Version 10, Philips Healthcare). The tool makes use of a threshold-based emphysema calculation approach applying a threshold of -950 Hounsfield units (HU). Insufficiently segmented lung volumes were manually reedited.

\subsection{ID Skewsness ${ }_{\text {PerfDef Emphysema-Index }}$}

The ID skewness in malperfused lung areas (ID Skewsness PerfDef $_{\text {f }}$-Emphysema-Index ( $\delta$-index) reflecting the ratio of ID distribution in malperfused lung areas and the proportion of emphysematous lung parenchyma was calculated forming the quotient of these two parameters. Discrete translation was

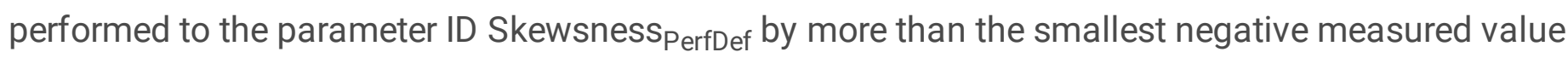
$(-1.35)$ to ensure that the data distribution was transformed in a strictly positive range. To obtain a calculable devisor, the proportion of emphysematous lung parenchyma was also transformed.

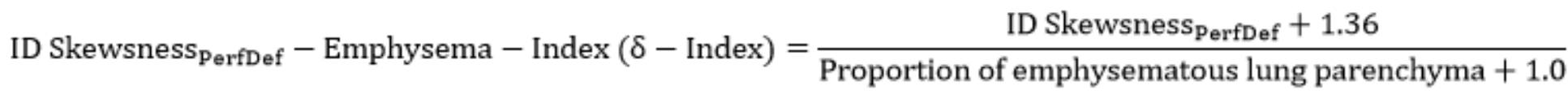

\subsection{Statistical analysis}

Normal distribution was tested using the Shapiro-Wilk test. Differences in continuous, parametric data were compared using the $t$-test. Continuous, independent, nonparametric data were compared using the Mann-Whitney $U$ test. Differences in categorical data were identified using Pearson's chi-squared test. Variances among and between the subgroups concerning continuous data were compared using ANOVA for parametric data. After assessing the equality of variances using Leven's test, post hoc testing was performed by Bonferroni adjustment for multiple comparisons. On the other hand, differences between the subgroups for categorial, nonparametric variables were assessed using the Kruskal-Wallis test and Dunn-Bonferroni corrected post hoc analysis. The area under the receiver operating characteristic curve (AUC) was calculated for subclassification. Based on AUC analysis parameters, sensitivity and specificity for subclassification were calculated using Youden's index.

A p-value of $<0.05$ was considered statistically significant. Statistical analysis was performed using SPSS software (IBM SPSS Statistics for macOS, Version 27.0, Armonk, NY, USA).

\section{Results}

\subsection{Study population}

All patient demographics are given in Table 1. 201 patients were screened for $\mathrm{PH}$. Of these, three patients were not eligible for $\mathrm{RHC}$, and 14 had an inconclusive $\mathrm{RHC}$ or too low pulmonary arterial contrast for image analyses, defined by an ID in the MPA $<5 \mathrm{mg} / \mathrm{mL}$. Subgroup 5 was excluded because of its small 
sample size $(n=2)$. For 20 patients, $R H C$ ruled out $P H(m P A P<25 \mathrm{mmHg})$, and these patients served as controls (Fig. 2). CTPA was performed a median of one day after RHC (min: 0 days, max: 4 days).

Table 1

Baseline characteristics

\begin{tabular}{|c|c|c|c|}
\hline & $\mathrm{PH}$ & Controls & $p$ \\
\hline Parameter & $(n=162)$ & $(n=20)$ & \\
\hline Age [years] & $70(57-77)$ & $64(57-78)$ & 0.82 \\
\hline $\operatorname{Sex}[\mathrm{m} / \mathrm{f}]$ & $60 / 102$ & $7 / 13$ & 0.12 \\
\hline NYHA class & & & 0.32 \\
\hline 1 & 1 & 0 & \\
\hline 2 & 17 & 3 & \\
\hline 3 & 102 & 14 & \\
\hline 4 & 13 & 0 & \\
\hline NT-pro BNP & $918(303-2160)$ & $338(154-620)$ & $<0.01$ \\
\hline \multicolumn{4}{|l|}{ RHC } \\
\hline RA pressure $[\mathrm{mmHg}]$ & $8.0(5.0-11.0)$ & $4.0(2.0-7.0)$ & 0.001 \\
\hline SvO2 [\%] & $64.3(59.8-68.2)$ & $67.8(63.1-71.0)$ & 0.04 \\
\hline $\mathrm{Cl}\left[\mathrm{l} / \mathrm{min} / \mathrm{m}^{2}\right]$ & $2.2(1.9-2.6)$ & $2.4(2.1-3.0)$ & 0.049 \\
\hline $\mathrm{mPAP}[\mathrm{mmHg}]$ & $40.5(32.8-50.0)$ & $21.0(18.3-23.0)$ & $<0.001$ \\
\hline $\mathrm{PVR}^{\star}[\mathrm{mmHg}]$ & $6.1(3.9-9.6)$ & $2.1(1.5-2.6)$ & $<0.001$ \\
\hline PCWP [mmHg] & $14.0(10.0-18.3)$ & $10.5(9.0-12.0)$ & 0.01 \\
\hline \multicolumn{4}{|l|}{ Echocardiography } \\
\hline $\mathrm{RA}$ area $\left[\mathrm{cm}^{2}\right]$ & $22.0(19.0-28.6)$ & $22.0(17.0-27.9)$ & 0.42 \\
\hline TAPSE [mm] & $20.0(16.1-23.0)$ & $22.0(18.0-24.0)$ & 0.25 \\
\hline \multicolumn{4}{|c|}{ Data are given as the mean (IQR). } \\
\hline \multicolumn{4}{|c|}{$\begin{array}{l}\text { PH, pulmonary hypertension; NYHA class, New York Heart Association (NYHA) Functional } \\
\text { Classification;38]; NT-pro BNP; N-terminal pro-B-type natriuretic peptide; RHC, right heart catherization } \\
\text { RA, right atrium; SvO2, pulmonary arterial oxygen saturation }\left(\mathrm{SvO}^{2}\right) \text {; } \mathrm{Cl} \text {, cardiac index; mPAP, mean } \\
\text { pulmonary artery pressure; PVR, pulmonary vascular resistance; PCWP, pulmonary capillary wedge } \\
\text { pressure; TAPSE, tricuspid annular plane systolic excursion. *defined as: (mPAP - PCWP)/cardiac } \\
\text { output }\end{array}$} \\
\hline
\end{tabular}




\subsection{CTPA-based differentiation between PH patients and healthy controls}

Table 2 displays all CTPA-based lung perfusion parameters in normal and malperfused lung areas for patients with PH and controls. Based on the ID in the MPA and the LA, right-to-left-heart contrast transit via the pulmonary vasculature was similar between groups. Patients suffering from $\mathrm{PH}$ showed higher proportions of malperfused lung areas $(p=0.02)$ as well as stronger (mean ID, $p=0.03$ ) and more homogenous perfusion deficits (ID skewness, $p=0.03$ ). Neither ID skewness in normal perfused lung areas nor ID kurtosis in any lung compartment differed significantly between groups.

Table 2

CTPA-based lung perfusion in patients and controls

\begin{tabular}{|c|c|c|c|}
\hline & $\mathrm{PH}$ & Controls & \multirow[t]{2}{*}{$p$} \\
\hline Parameter & $(n=162)$ & $(n=20)$ & \\
\hline ID MPA & $14.1(11.6-16.9)$ & $15.1(11.7-17.1)$ & 0.93 \\
\hline ID LA & $8.9(7.2-10.5)$ & $9.6(8.4-12.4)$ & 0.07 \\
\hline \multicolumn{4}{|l|}{ Normal perfused lung } \\
\hline Volume [\% of total lung volume] & $57.1(37.5-73.6)$ & $72.6(55.9-79.9)$ & 0.01 \\
\hline Mean ID & $0.091(0.083-0.109)$ & $0.095(0.085-0.121)$ & 0.52 \\
\hline ID Kurtosis & $4.67(2.45-7.42)$ & $6.77(2.63-9.72)$ & 0.16 \\
\hline ID Skewness & $1.98(1.63-2.50)$ & $2.43(1.42-2.84)$ & 0.27 \\
\hline \multicolumn{4}{|l|}{ Malperfused lung } \\
\hline Volume [\% of total lung volume] & $39.0(24.0-59.7)$ & $25.6(16.8-42.7)$ & 0.02 \\
\hline Mean ID & $0.026(0.021-0.030)$ & $0.028(0.025-0.032)$ & 0.03 \\
\hline ID Kurtosis & $-1.00(-1.25--0.62)$ & $-1.11(-1.33--0.16)$ & 0.77 \\
\hline ID Skewness & $-0.30(-0.62-0.08)$ & $-0.47(-0.85--0.26)$ & 0.03 \\
\hline \multicolumn{4}{|l|}{ Data are given as the mean (IQR). } \\
\hline
\end{tabular}

\subsection{CTPA-based differentiation of PH subgroups}

The Kruskal-Wallis test revealed significant differences between $\mathrm{PH}$ subgroups for the percentage of normal perfused lung volume $\left(\mathrm{V}_{\text {PerfNorm }}\right)$ and malperfused lung volume $\left(\mathrm{V}_{\text {PerfDef }}\right)$, mean ID, ID kurtosis as well as ID skewness in malperfused lung areas ( $p$ for all < 0.001) (Table 3$)$. 
Table 3

CTPA-based pulmonary perfusion and pulmonary parenchyma characteristics in different PH subgroups Nice class

\begin{tabular}{lllllll} 
& $\mathbf{1}$ & $\mathbf{2}$ & $\mathbf{3}$ & $\mathbf{4}$ & $\mathbf{c p c}$ & $\mathrm{p}$ \\
Parameter & $\mathbf{( n = 3 9 )}$ & $\mathbf{( n = 1 5 )}$ & $\mathbf{( n = 2 4 )}$ & $\mathbf{( n = 5 2 )}$ & $\mathbf{( n = 3 2 )}$ & \\
$\begin{array}{l}\text { Normal perfused } \\
\text { lung }\end{array}$ & & & & & & \\
Volume [\% of total & 62.9 & 75.9 & 42.2 & 55.4 & 57.6 & < \\
lung volume] & $(41.1-$ & $(64.8-$ & $(20.9-$ & $(39.2-$ & $(39.5-$ & $\mathbf{0 . 0 0 1 *}$ \\
& $78.2)$ & $87.7)$ & $58.9)$ & $70.4)$ & $70.4)$ & \\
\hline Mean ID & 0.090 & 0.115 & 0.092 & 0.093 & 0.089 & 0.48 \\
& $(0.081-$ & $(0.080-$ & $(0.085-$ & $(0.085-$ & $(0.083-$ & \\
& $0.104)$ & $0.135)$ & $0.112)$ & $0.111)$ & $0.100)$ & \\
ID Kurtosis & 5.03 & 4.51 & 3.64 & 4.56 & 5.22 & 0.84 \\
& $(2.43-$ & $(3.45-$ & $(2.34-$ & $(3.01-$ & $(2.35-$ & \\
& $6.77)$ & $9.49)$ & $7.14)$ & $7.40)$ & $8.19)$ & \\
ID Skewness & 2.15 & 1.84 & 1.86 & 1.90 & 2.10 & 0.88 \\
& $(1.63-$ & $(1.67-$ & $(1.59-$ & $(1.57-$ & $(1.62-$ & \\
& $2.64)$ & $2.68)$ & $2.48)$ & $2.44)$ & $2.66)$ & \\
\end{tabular}

Malperfused lung

\begin{tabular}{|c|c|c|c|c|c|c|}
\hline $\begin{array}{l}\text { Volume [\% of total } \\
\text { lung volume] }\end{array}$ & $\begin{array}{l}33.7 \\
(18.3- \\
56.6)\end{array}$ & $\begin{array}{l}21.4(7.7- \\
31.1)\end{array}$ & $\begin{array}{l}53.7 \\
(37.2- \\
77.9)\end{array}$ & $\begin{array}{l}42.5 \\
(27.8- \\
59.1)\end{array}$ & $\begin{array}{l}37.2 \\
(26.7- \\
58.8)\end{array}$ & <.001+ \\
\hline Mean ID & $\begin{array}{l}0.029 \\
(0.026- \\
0.033)\end{array}$ & $\begin{array}{l}0.029 \\
(0.025- \\
0.032)\end{array}$ & $\begin{array}{l}0.021 \\
(0.017- \\
0.025)\end{array}$ & $\begin{array}{l}0.023 \\
(0.020- \\
0.027)\end{array}$ & $\begin{array}{l}0.028 \\
(0.024- \\
0.031)\end{array}$ & $\dot{0} 001$ \\
\hline ID Kurtosis & $\begin{array}{l}-0.77 \\
(-0.98- \\
-0.16)\end{array}$ & $\begin{array}{l}-0.98 \\
(-1.28- \\
-0.05)\end{array}$ & $\begin{array}{l}-1.21 \\
(-1.39- \\
-0.93)\end{array}$ & $\begin{array}{l}-1.14 \\
(-1.26- \\
-0.97)\end{array}$ & $\begin{array}{l}-0.85 \\
(-1.21- \\
-0.35)\end{array}$ & $\hat{0} .001 \neq$ \\
\hline ID Skewness & $\begin{array}{l}-0.58 \\
(-0.87- \\
-0.13)\end{array}$ & $\begin{array}{l}-0.57 \\
(-0.85- \\
-0.22)\end{array}$ & $\begin{array}{l}0.03 \\
(-0.23- \\
0.54)\end{array}$ & $\begin{array}{l}-0.05 \\
(-0.33- \\
0.18)\end{array}$ & $\begin{array}{l}-0.47 \\
(-0.74- \\
-0.19)\end{array}$ & $\begin{array}{l}< \\
0.001\end{array}$ \\
\hline $\begin{array}{l}\text { Emphysema [\% of } \\
\text { total lung volume] }\end{array}$ & $\begin{array}{l}0.2(0.0- \\
1.8)\end{array}$ & $\begin{array}{l}0.1(0.1- \\
0.2)\end{array}$ & $\begin{array}{l}8.4(2.8- \\
17.1)\end{array}$ & $\begin{array}{l}0.4(0.1- \\
1.3)\end{array}$ & $\begin{array}{l}0.3(0.1- \\
2.0)\end{array}$ & ¿.001§ \\
\hline
\end{tabular}

Data are given as the mean (IQR).

CTPA, CT pulmonary angiography; cpc, combined pre- and postcapillary pulmonary hypertension; ID, iodine density. ${ }^{*} 3-2, p<0.001 ; 4-2, p=0.02,+3-2, p<0.001 ; 4-2, p=0.01, \neq 3-1, p<0,01 ; 4-1, p$ $<0.01$, § $3-1 ; 3-2 ; 3-4 ; 3-6 ; p$ for all $<0.001, \| 3-2, p<0.001,3-4, p<0.001 ; 4-1, p<0.01 ; 4-6, p$ $<0.01$. 


\section{Nice class}

$\begin{array}{lllllll}\text { S-index } & 0.43 & 0.72 & 0.17 & 0.75 & 0.44 & < \\ & (0.27- & (0.42- & (0.10- & (0.55- & (0.20- & 0.001 \| \\ & 0.66) & 1.03) & 0.34) & 1.10) & 0.62) & \end{array}$

Data are given as the mean (IQR).

CTPA, CT pulmonary angiography; cPc, combined pre- and postcapillary pulmonary hypertension; ID, iodine density. ${ }^{*} 3-2, p<0.001 ; 4-2, p=0.02,+3-2, p<0.001 ; 4-2, p=0.01, \neq 3-1, p<0,01 ; 4-1, p$ $<0.01$, § $3-1 ; 3-2 ; 3-4 ; 3-6 ; p$ for all $<0.001, \| 3-2, p<0.001,3-4, p<0.001 ; 4-1, p<0.01 ; 4-6, p$ $<0.01$.

Patients suffering from $\mathrm{PH}$ due to chronic lung disease and/or hypoxia had the largest extent of $\mathrm{V}_{\text {PerfDef }}$ among all subgroups, with a significantly higher proportion of malperfused lung areas compared to Nice class 2 (Table 3). Moreover, malperfused lung areas showed a significantly stronger (mean ID) and more homogenous perfusion deficit (ID skewness) compared to all other subgroups ( $p$ for all $<0.001$, Fig. 3 ), except for subgroup 4 ( $p=1.00$, respectively).

Corresponding results were demonstrated for patients with CTEPH showing a significantly stronger perfusion deficit as well as more homogenous ID distribution in malperfused lung areas compared to all other subgroups ( $p$ for all $<0.001$, only for mean ID 4-6 $p=0.01$, Fig. 3 ), except subgroup $3(p=1.00)$. ID skewness in malperfused lung areas allowed for an accurate identification of subgroups 3 and 4 based on AUC analysis (AUC: $0.79,95 \%-\mathrm{Cl}, 0.72-0.86$ ). Applying a cut-off of -0.335 as determined by Youden's index, a sensitivity of $84 \%$ and a specificity of $70 \%$ could be achieved for subclassification. Figure 4 illustrates the difference in pattern and ID distribution of malperfused lung areas in patients with PAH and patients suffering from CTEPH.

Neither normal nor malperfused lung areas revealed any difference between subgroups 1 and 2 and patients suffering from cpc.

The $\delta$-index differed significantly between subgroups 3 and 4 (Table 3, Fig. 5). Based on AUC analysis, the index allowed for an accurate identification of patients with CTEPH (AUC: $0.92,95 \%-\mathrm{Cl}, 0.85-0.99$ ). A cut-off of 0.502 as determined by Youden's index was best for subclassification (sensitivity $86 \%$, specificity $85 \%$ ). The stepwise differentiation of both subgroups based on IDIs and VNC-based lung emphysema quantification is exemplified in Fig. 6.

\section{Discussion}

With the to our knowledge, the first study evaluating the potential of SDCT-derived pulmonary perfusion maps and pulmonary parenchyma characteristics for the semiautomated diagnosis and classification of $\mathrm{PH}$ with differing etiologies we can present several notable findings. 1. Semiautomatic lung segmentation into normal and malperfused lung areas based on ID values is feasible and applicable in the setting of $\mathrm{PH}$. 2. The proportion of normal to malperfused lung areas and the mean ID and ID skewness in malperfused lung parenchyma can aid the identification of patients with $\mathrm{PH}$. 3. ID skewness in 
malperfused lung areas and the introduced $\delta$-index are powerful parameters for the identification of $\mathrm{PH}$ subgroups, especially for subclassification Nice classes 3 and 4 .

\subsection{Differentiation of PH patients and healthy controls}

The accurate diagnosis of $\mathrm{PH}$ remains challenging, 28], and no meaningful decrease in the time from symptom onset to diagnosis could be achieved throughout the last decades.24]. This also accounts for the high morbidity and mortality of $\mathrm{PH}$, regardless of the underlying etiology.2-5]. Although $\mathrm{RHC}$ is accompanied by peri- and postinterventional complications and does not allow for morphologic information, 29], it remains the diagnostic gold standard, 1], as noninvasive imaging parameters either lack diagnostic accuracy $28,30,31$ ] or have not yet been validated in large study cohorts, albeit promising initial results.32, 33]. This is in line with our findings: The extent of perfusion defects and the ID skewness in malperfused lung areas differed significantly between patients with $\mathrm{PH}$ and controls and thus might aid the detection of disease. However, subgroup analysis revealed no significant difference in any volumetric pulmonary perfusion parameter between Nice classes 1 and 2 and controls ( $p$ for all > 0.05 , data not shown). Concurrently, Kim et al. found only $60.0 \%$, and Giordano et al. even found only $52.6 \%$ of PAH patients showing abnormal pulmonary perfusion.19, 20]. Although it remains unclear to what extent our results might be affected by the composition of the control group, which consisted of patients with the clinical suspicion of $\mathrm{PH}$ and a borderline mPAP of $21.0 \mathrm{mmHg}$, our data thus underscore the challenges of a noninvasive $\mathrm{PH}$ diagnosis.

\subsection{Classification of PH subgroups}

Our results demonstrate the merit of SDCT-derived pulmonary perfusion maps and pulmonary parenchyma characteristics over and above RHC for the classification of $\mathrm{PH}$ subgroups, particularly the spectrum of precapillary $\mathrm{PH}$, which remains the main task from the radiologist's view.20].

There is evidence from V/Q-scintigraphic and DECT-based studies that pulmonary perfusion maps might enable the differentiation of $\mathrm{PH}$ subgroups, as different types of perfusion abnormalities correlate with PH etiology.18-21]. Regardless of the modality, these studies are limited due to small sample sizes and/or time-consuming quantitative or semiquantitative image interpretation with considerable intra- and interreader variability. We demonstrated that semiautomatically generated volumetric parameters of SDCT-based pulmonary perfusion maps can aid the classification of $\mathrm{PH}$. On the basis of ID skewness in malperfused lung areas, patients suffering from $\mathrm{PH}$ due to chronic lung disease and/or hypoxia and patients with CTEPH could confidently be differentiated from all other subgroups. Corresponding to the characteristic multisegmental, sharply defined, wedge-shaped and hypoattenuated appearance, 15, 34], which is also described for PE, 10], malperfused lung areas in patients with CTEPH stood out due to more pronounced and more homogenous perfusion defects. The same was true for patients with PH due to chronic lung disease and/or hypoxia, which can be explained by the emphysematous lung changes prevalent in this patient group leading to large areas with no iodine attenuation at all. 
Together with subgroups 1 and 5, subgroups 3 and 4 form the spectrum of precapillary PH. The subclassification of precapillary $\mathrm{PH}$ marks a key point in the diagnostic work-up of $\mathrm{PH}$, as the distinct forms cannot be differentiated via RHC.1]. In this context, the identification of patients with CTEPH plays a pivotal role since these patients face a particularly poor prognosis 35] and can potentially be cured by surgical thrombendarteriectomy or balloon angioplasty. The evaluation of DECT-derived pulmonary perfusion maps for the diagnosis of CTEPH has been validated in numerous studies against V/Qscintigraphy, 14, 15], which is considered the gold standard to screen for the disease.1]. With the introduction of the SDCT-based $\delta$-index, we were able to identify a powerful parameter that allows for the semiautomated identification of patients with CTEPH. Contrary to previous SDCT studies differentiating CTEPH from other forms of $\mathrm{PH}, 14,15,21]$, this approach works independently of reader expertise. This might be helpful to facilitate confident diagnosis of CTEPH for radiologists with limited expertise in $\mathrm{PH}$ imaging. In comparison to V/Q-scintigraphy, it overcomes the modality's inherent main limitation of V/Qscintigraphy, which is unable to obtain morphologic information such as parenchymal changes or vessel anatomy.11, 14, 15, 17]. In combination with the interpretation of the pulmonary vasculature, SDCT-based pulmonary perfusion maps might thus aid the identification of patients with CTEPH and a high probability of profiting from treatment.20]. Unlike a sequential approach of V/Q-scintigraphy and conventional CTA, the integrated SDCT approach offers the unique possibility of quantifying parenchymal lung disease based on VNC images without the necessity of extra radiation exposure.22, 36]. The $\delta$-index thus automatically accounts for the radiologist's task to interpret iodine maps in correlation with pulmonary parenchyma alterations to differentiate between true perfusion defects and perfusion defects due to pulmonary pathologies such as emphysema.35].

\subsection{Limitations}

Apart from the retrospective study design, several limitations need to be addressed. First, the inability of the $\delta$-index to differentiate between true perfusion defects and pseudodefects, e.g., due to beam hardening or motion artifacts, is a considerable limitation.37]. Second, due to scan timing, SDCT-derived perfusion maps only provide very limited information on lung compartments with maintained blood supply beyond the occluded pulmonary arteries through systemic collateral blood flow, e.g., via bronchial arteries, best described for CTEPH, 35], and thus might overestimate the degree of perfusion deficit. This probably represents the methods biggest limitation. Third, we did not differentiate between major-vessel and minor-vessel CTEPH, which is known to partly mimic typical PAH perfusion deficit patterns.20]. Validation of our findings in a PAH and subclassified CTEPH population thus seems highly desirable. Last, our data did not allow for an independent validation of the VNC-based emphysema quantification, as the CTPA protocol did not include true noncontrast images. Further studies addressing this considerable limitation are highly warranted.

\subsection{Conclusion}

SDCT-derived pulmonary perfusion and pulmonary parenchyma characteristics can detect and quantify pulmonary perfusion abnormalities in $\mathrm{PH}$ and allow for a semiautomated diagnosis of Nice classes 3 and 4 , independent of reader expertise. 


\section{Abbreviations}

\begin{tabular}{ll} 
CO & Cardiac output \\
\hline CTEPH & Chronic thromboembolic pulmonary hypertension \\
\hline CTPA & CT pulmonary angiography \\
\hline DECT & Dual energy CT \\
\hline ID & lodine density \\
\hline IDI & lodine density image \\
\hline LA & Left atrium \\
\hline MPA & Mean pulmonary artery \\
\hline mPAP & Mean pulmonary artery pressure \\
\hline PAH & Pulmonary arterial hypertension \\
\hline PCWP & Pulmonary capillary wedge pressure \\
\hline PH & Pulmonary hypertension \\
\hline PVR & Pulmonary vascular resistance \\
\hline RA & Right atrial \\
\hline RHC & Right heart catheterization \\
\hline SDCT & Spectral detector CT \\
\hline SVO & Pulmonary arterial oxygen saturation \\
\hline TTE & Transthoracic echocardiography \\
\hline VNC & Virtual non-contrast \\
\hline
\end{tabular}

\section{Declarations}

\section{Data availability:}

The data that support the findings of this study are available from the corresponding author upon reasonable request. The corresponding author had full access to all the data in the study and takes responsibility for its integrity and the data analysis.

\section{Additional information:}

Competing interests: 
Roman J. Gertz: Received research support from Philips Healthcare. Nils Große Hokamp has been supported by the Else Kröner-Fresenius-Stiftung (2018_EKMS.34 to Nils Große Hokamp) and receives research support from Philips Healthcare. Nils Große Hokamp and David Maintz are on the speaker's bureau of Philips Healthcare.

All other authors declare no potential conflicts of interest.

\section{Authors contributions statement:}

Data collection, image analysis, statistical analysis and the writing of the manuscript, including graphic preparation, were performed by Roman Johannes Gertz. Felix Gerhardt and Stephan Rosenkranz carried out invasive testing. Rahil Shahzad and Liliana Caldeira performed image feature extraction and analysis. Jan Robert Kröger supported image analysis and study design. Jonathan Kottlors supported statistical analysis. Nils Große Hokamp and David Maintz supported graphic preparation and manuscript conceptualization. Alexander Christian Bunck created the study design, supported graphic preparation and manuscript conceptualization and carried out project supervision. All authors reviewed the manuscript.

\section{References}

1. Galiè, N. et al. 2015 ESC/ERS guidelines for the diagnosis and treatment of pulmonary hypertension: the joint task force for the diagnosis and treatment of pulmonary hypertension of the European Society of Cardiology (ESC) and the European Respiratory Society (ERS): Endor. Eur Heart J, 37, 67119 https://doi.org/10.1093/eurheartj/ehv317 (2016).

2. Coghlan, J. G. \& Handler, C. Connective tissue associated pulmonary arterial hypertension. Lupus, 15, 138-142 https://doi.org/10.1191/0961203306lu2280rr (2006).

3. Lettieri, C. J. et al. Prevalence and outcomes of pulmonary arterial hypertension in advanced idiopathic pulmonary fibrosis., 129, 746-752 https://doi.org/10.1378/chest.129.3.746 (2006).

4. Nunes, H. et al. Pulmonary hypertension associated with sarcoidosis: mechanisms, haemodynamics and prognosis., 61, 68-74 https://doi.org/10.1136/thx.2005.042838 (2006).

5. Rubin, L. J. Diagnosis and management of pulmonary arterial hypertension: ACCP evidence-based clinical practice guidelines., 126, https://doi.org/10.1378/chest.126.1_suppl.7S (2004). 7S-10S

6. Thieme, S. F. et al. Dual-energy CT for the assessment of contrast material distribution in the pulmonary parenchyma. AJR Am J Roentgenol, 193, 144-149 https://doi.org/10.2214/AJR.08.1653 (2009).

7. Stiller, W. et al. Correlation of quantitative dual-energy computed tomography iodine maps and abdominal computed tomography perfusion measurements: are single-acquisition dual-energy computed tomography iodine maps more than a reduced-dose surrogate of conventional computed tomography perfusion? Invest Radiol, 50, 703-708 https://doi.org/10.1097/RLI.0000000000000176 (2015). 
8. Skornitzke, S. et al. Dual-energy CT iodine maps as an alternative quantitative imaging biomarker to abdominal CT perfusion: determination of appropriate trigger delays for acquisition using bolus tracking. Br J Radiol, 91, 20170351 https://doi.org/10.1259/bjr.20170351 (2018).

9. Ferda, J. et al. Pulmonary imaging using dual-energy CT, a role of the assessment of iodine and air distribution. Eur J Radiol, 77, 287-293 https://doi.org/10.1016/j.ejrad.2009.08.005 (2011).

10. Pontana, F. et al. Lung perfusion with dual-energy multidetector-row CT (MDCT): feasibility for the evaluation of acute pulmonary embolism in 117 consecutive patients. Acad Radiol, 15, 1494-1504 https://doi.org/10.1016/j.acra.2008.05.018 (2008).

11. Thieme, S. F. et al. Dual energy CT for the assessment of lung perfusion-correlation to scintigraphy. Eur J Radiol, 68, 369-374 https://doi.org/10.1016/j.ejrad.2008.07.031 (2008).

12. Zhang, L-J. et al. Detection of pulmonary embolism by dual energy CT: correlation with perfusion scintigraphy and histopathological findings in rabbits. Eur Radiol, 19, 2844-2854 https://doi.org/10.1007/s00330-009-1518-z (2009).

13. Bauer, R. W. et al. Lung perfusion analysis with dual energy CT in patients with suspected pulmonary embolism-Influence of window settings on the diagnosis of underlying pathologies of perfusion defects. Eur J Radiol, 80, e476-e482 https://doi.org/10.1016/j.ejrad.2010.09.009 (2011).

14. Dournes, G. et al. Dual-energy CT perfusion and angiography in chronic thromboembolic pulmonary hypertension: diagnostic accuracy and concordance with radionuclide scintigraphy. Eur Radiol, 24, 42-51 https://doi.org/10.1007/s00330-013-2975-y (2014).

15. Masy, M. et al. Dual-energy CT (DECT) lung perfusion in pulmonary hypertension: concordance rate with V/Q scintigraphy in diagnosing chronic thromboembolic pulmonary hypertension (CTEPH). Eur Radiol, 28, 5100-5110 https://doi.org/10.1007/s00330-018-5467-2 (2018).

16. Okada, M. et al. added value of lung perfused blood volume images using dual-energy CT for assessment of acute pulmonary embolism. Eur J Radiol, 84, 172-177 https://doi.org/10.1016/j.ejrad.2014.09.009 (2015).

17. Nakazawa, T. et al. Lung perfused blood volume images with dual-energy computed tomography for chronic thromboembolic pulmonary hypertension: correlation to scintigraphy with single-photon emission computed tomography. J Comput Assist Tomogr, 35, 590-595 https://doi.org/10.1097/RCT.0b013e318224e227 (2011).

18. Lisbona, R., Kreisman, H., Novales-Diaz, J. \& Derbekyan, V. Perfusion lung scanning: differentiation of primary from thromboembolic pulmonary hypertension. AJR Am J Roentgenol, 144, 27-30 https://doi.org/10.2214/ajr.144.1.27 (1985).

19. Kim, E. Y. et al. Assessment of perfusion pattern and extent of perfusion defect on dual-energy CT angiography: correlations between the causes of pulmonary hypertension and vascular parameters. Korean J Radiol, 15, 286-294 https://doi.org/10.3348/kjr.2014.15.2.286 (2014).

20. Giordano, J. et al. Lung perfusion characteristics in pulmonary arterial hypertension (PAH) and peripheral forms of chronic thromboembolic pulmonary hypertension (pCTEPH): Dual-energy CT 
experience in 31 patients. Eur Radiol, 27, 1631-1639 https://doi.org/10.1007/s00330-016-4500-6 (2017).

21. Kröger, J. R. et al. Diagnosis of pulmonary hypertension using spectral-detector CT. Int J Cardiol, 285, 80-85 https://doi.org/10.1016/j.ijcard.2019.03.018 (2019).

22. Wook Lee, C. et al. A pilot trial on pulmonary emphysema quantification and perfusion mapping in a single-step using contrast-enhanced dual-energy computed tomography. Invest Radiol, 47 (1), 92-97 https://doi.org/10.1097/RLI.0b013e318228359a (2012).

23. Lu, G. M., Zhao, Y., Zhang, L. J. \& Schoepf, U. J. Dual-energy CT of the lung. AJR Am J Roentgen, 199, S40-53 https://doi.org/10.2214/AJR.12.9112 (2012).

24. Frost, A. et al. Diagnosis of pulmonary hypertension. Eur Respir J, 53, 1801904 https://doi.org/10.1183/13993003.01904-2018 (2019).

25. Große Hokamp, N. et al. Technical background of a novel detector-based approach to dual-energy computed tomography. Diagn Interv Radiol, 26, 68-71 https://doi.org/10.5152/dir.2019.19136 (2020).

26. Große Hokamp, N. et al. Assessment of arterially hyper-enhancing liver lesions using virtual monoenergetic images from spectral detector CT: phantom and patient experience. Abdom Radiol (NY), 43, 2066-2074 https://doi.org/10.1007/s00261-017-1411-1 (2018).

27. Zopfs, D. et al. Intraindividual consistency of iodine concentration in dual-energy computed tomography of the chest and abdomen. Invest Radiol, 56 (3), 181-187 https://doi.org/https:// (2021).

28. Shen, Y. et al. CT-base pulmonary artery measurement in the detection of pulmonary hypertension: a meta-analysis and systematic review., 93, e256-e256 https://doi.org/10.1097/MD.0000000000000256 (2014).

29. Hoeper, M. M. et al. Complications of right heart catheterization procedures in patients with pulmonary hypertension in experienced centers. J Am Coll Cardiol, 48, 2546-2552 https://doi.org/10.1016/j.jacc.2006.07.061 (2006).

30. Wang, N. et al. A systematic review of the diagnostic accuracy of cardiovascular magnetic resonance for pulmonary hypertension. Can J Cardiol, 30, 455-463 https://doi.org/10.1016/j.cjca.2013.11.028 (2014).

31. Janda, S., Shahidi, N., Gin, K. \& Swiston, J. Diagnostic accuracy of echocardiography for pulmonary hypertension: a systematic review and meta-analysis., 97, 612 LP 622https://doi.org/10.1136/hrt.2010.212084 (2011).

32. Pienn, M. et al. Non-invasive determination of pulmonary hypertension with dynamic contrastenhanced computed tomography: a pilot study. Eur Radiol, 24, 668-676 https://doi.org/10.1007/s00330-013-3067-8 (2014).

33. Revel, M. P. et al. Pulmonary Hypertension: ECG-gated 64-section CT angiographic evaluation of new functional parameters as diagnostic criteria., 250, 558-566 https://doi.org/10.1148/radiol.2502080315 (2009). 
34. Ameli-Renani, S. et al. Dual-Energy CT for imaging of pulmonary hypertension: challenges and opportunities., 34, 1769-1790 https://doi.org/10.1148/rg.347130085 (2014).

35. Kharat, A., Hachulla, A. L., Noble, S. \& Lador, F. Modern diagnosis of chronic thromboembolic pulmonary hypertension. Thromb Res, 163, 260-265 https://doi.org/10.1016/j.thromres.2017.09.008 (2018).

36. Pansini, V. et al. Assessment of lobar perfusion in smokers according to the presence and severity of emphysema: preliminary experience with dual-energy CT angiography. Eur Radiol, 19, 2834 https://doi.org/10.1007/s00330-009-1475-6 (2009).

37. Kang, M. J. et al. Focal iodine defects on color-coded iodine perfusion maps of dual-energy pulmonary CT angiography images: a potential diagnostic pitfall. AJR Am J Roentgen, 195, W32530 https://doi.org/10.2214/AJR.09.3241 (2010).

38. The Criteria Committee of the New York Heart Association (1994). Nomenclature and criteria for diagnosis of diseases of the heart and great vessels. 9th Edition, Little Brown \& Co., Boston, 253256

\section{Figures}

A
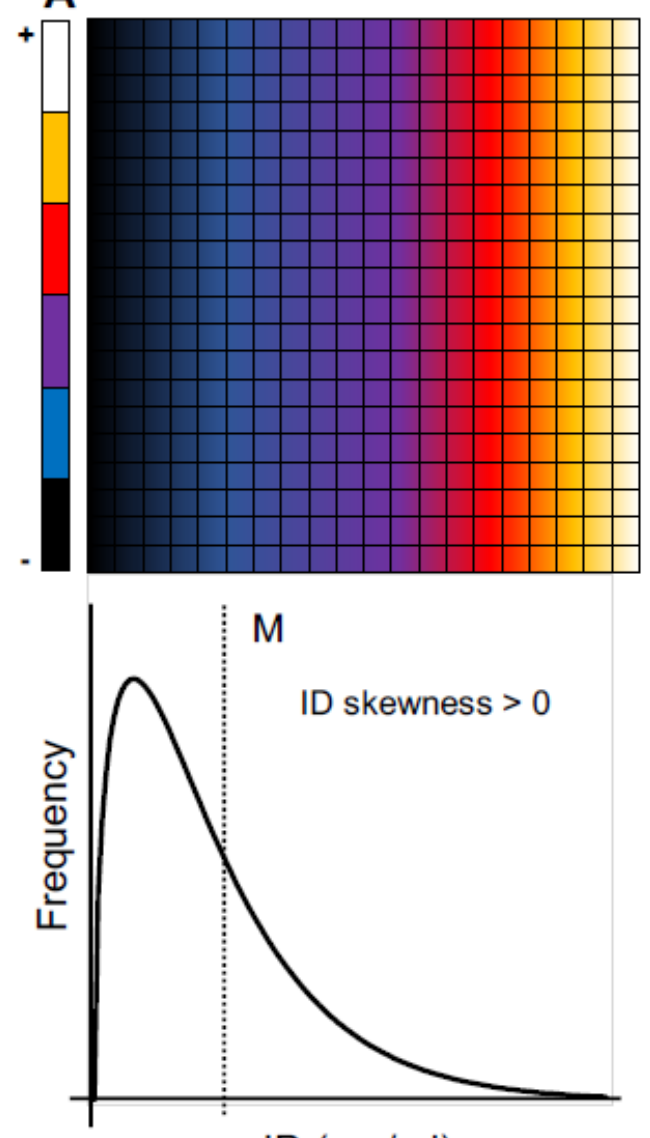

ID $(\mathrm{mg} / \mathrm{ml})$
B
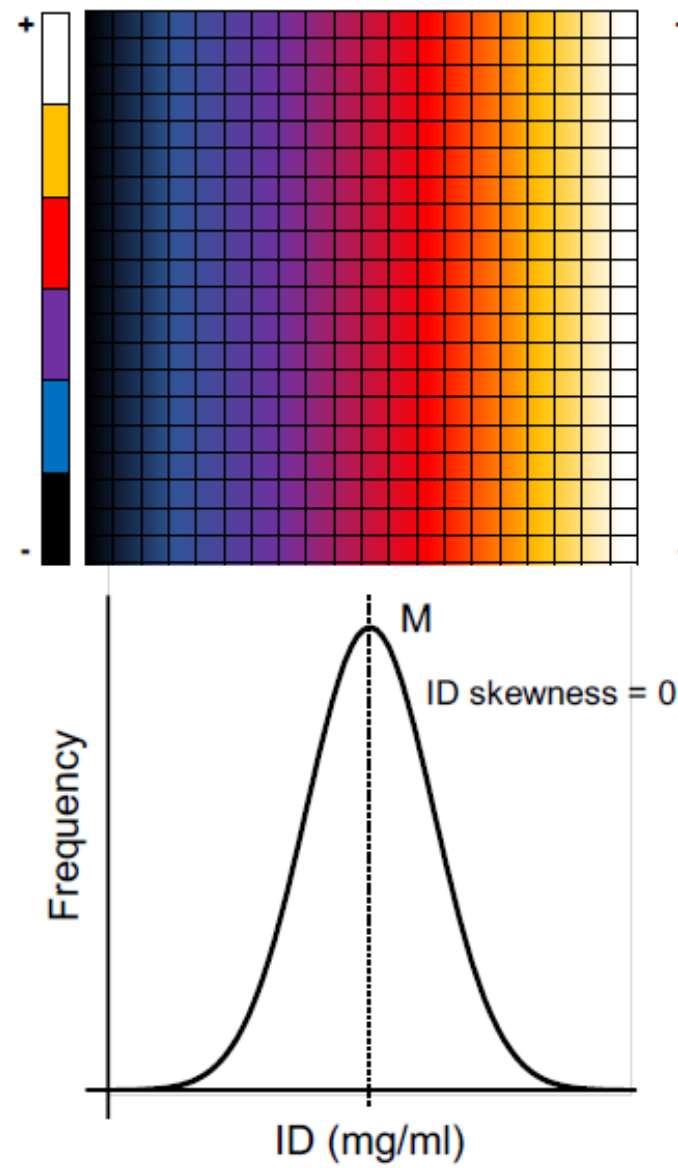
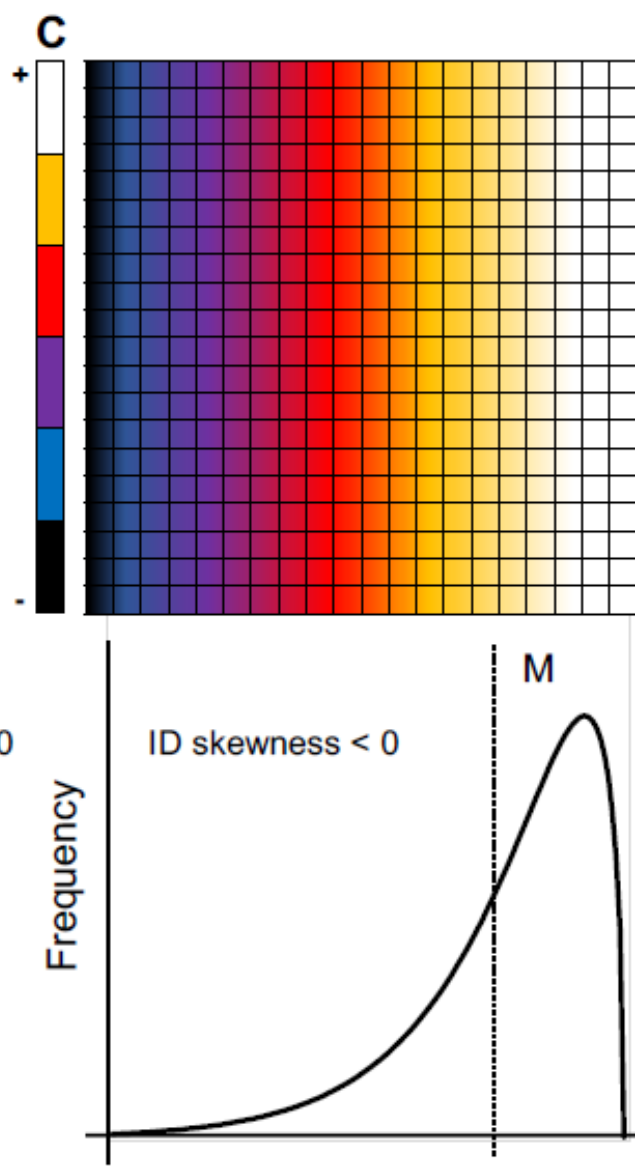

ID $(\mathrm{mg} / \mathrm{ml})$

Figure 1 
Different types of ID distributions and corresponding ID skewness. A majority of pixels/voxels with an ID below the mean result in a positively skewed ID distribution (ID skewness $>0$, Panel A), while a majority of pixels/voxels with an ID above the mean result in a negatively skewed ID distribution (ID skewness $<0$, Panel C). The more homogenous the ID distribution becomes (ultimately tending towards a normal distribution), the more the ID skewness approaches zero (ID skewness $=0$, Panel $B$ ). $M$, mean; ID, iodine density.

\section{1 patients screened for $\mathrm{PH}$}

no RHC $(n=3)$

\section{8 patients underwent RHC and CTPA}

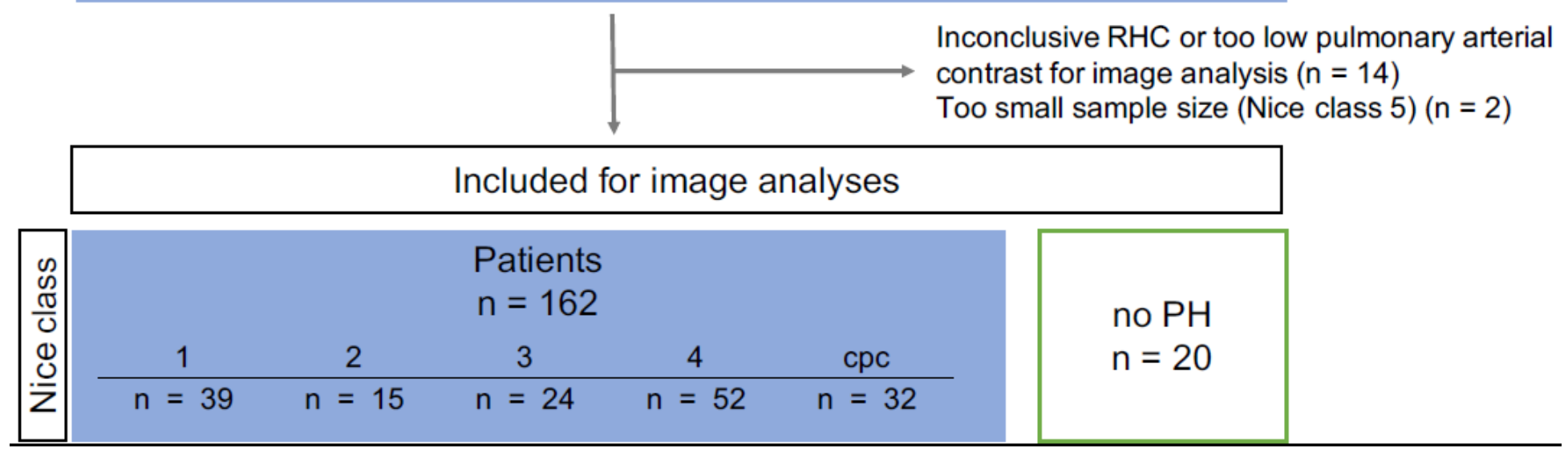

\section{Figure 2}

Study-flow-chart. PH, pulmonary hypertension; RHC, right heart catherization; CTPA, CT pulmonary angiography; cpc, combined pre- and postcapillary pulmonary hypertension. 

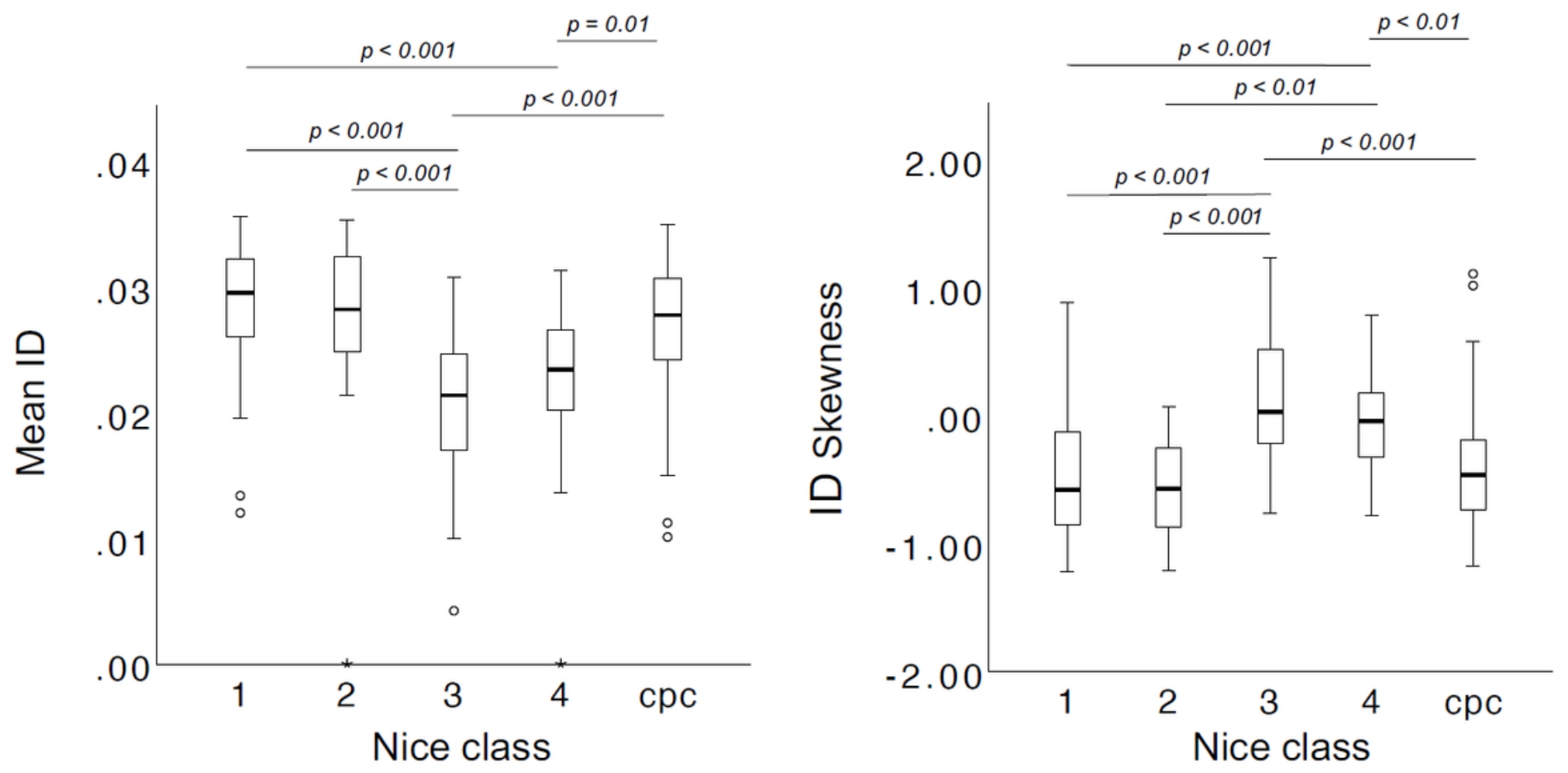

Figure 3

CTPA-based differentiation of PH subgroups according to mean ID and ID skewness in malperfused lung areas. ID, iodine density; cpc, combined pre- and postcapillary pulmonary hypertension. 

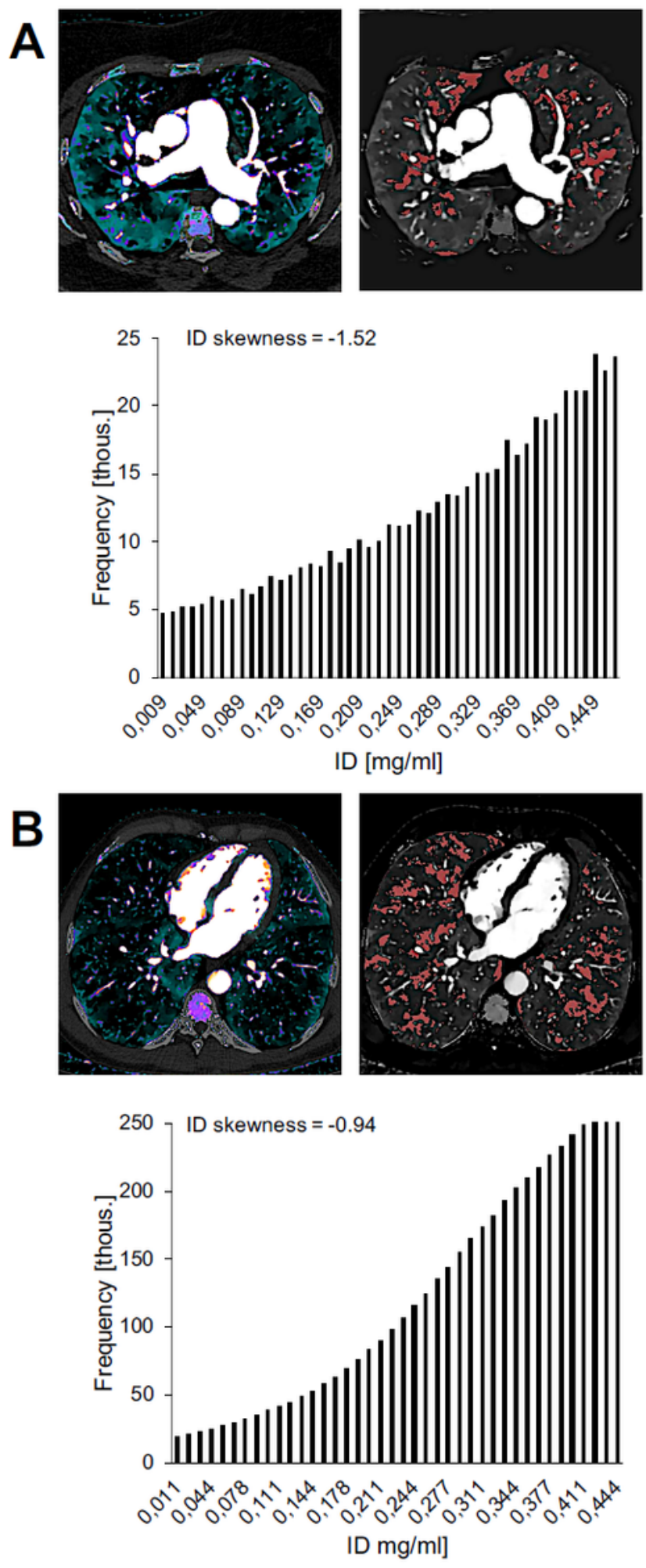
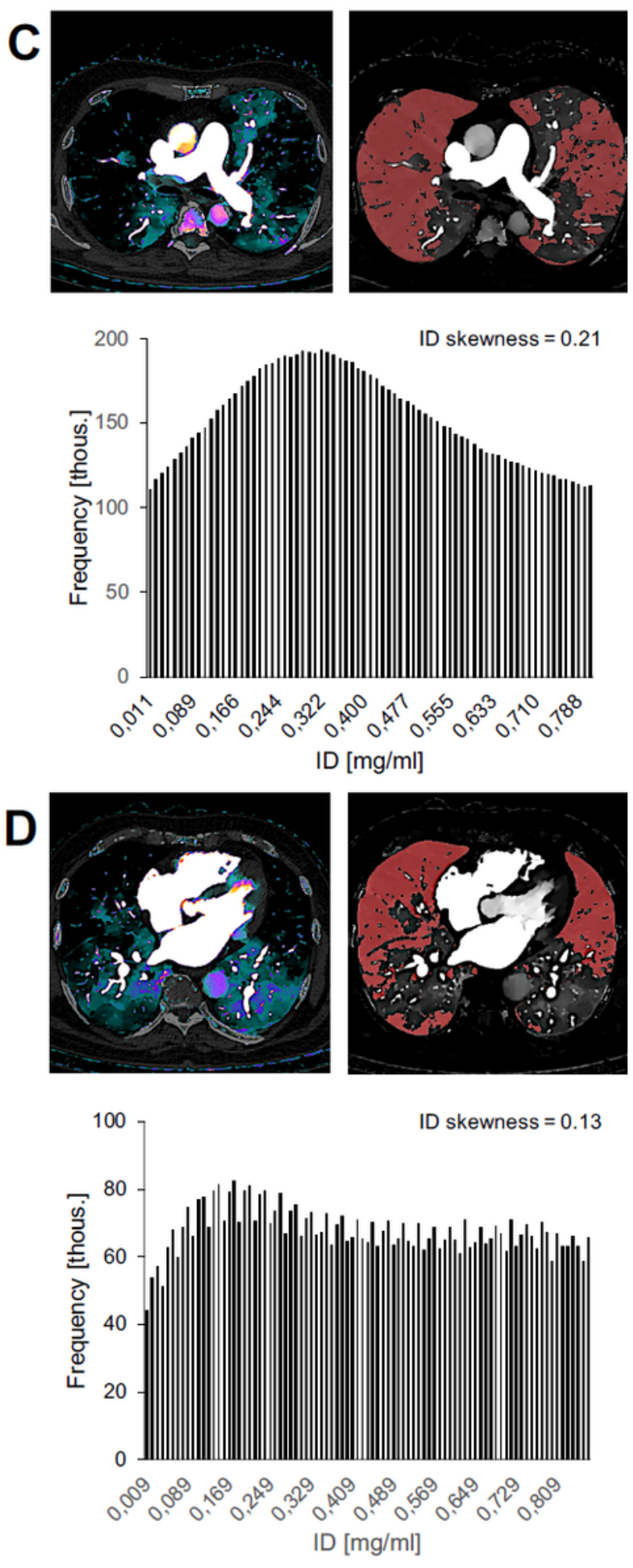

Figure 4

IDIs with automatically derived malperfused lung areas (red) and corresponding ID histograms of the perfusion deficit volume illustrating the difference of pattern and ID distribution of malperfused lung areas in two example cases of PAH (Panel A and B) and CTEPH (Panel C and D), respectively. Corresponding histograms indicate an inhomogeneous ID distribution in PAH (ID skewness < 0 ) and a more homogenous ID distribution in CTEPH (ID skewness close to 0). Thous., thousand; ID, iodine density. 

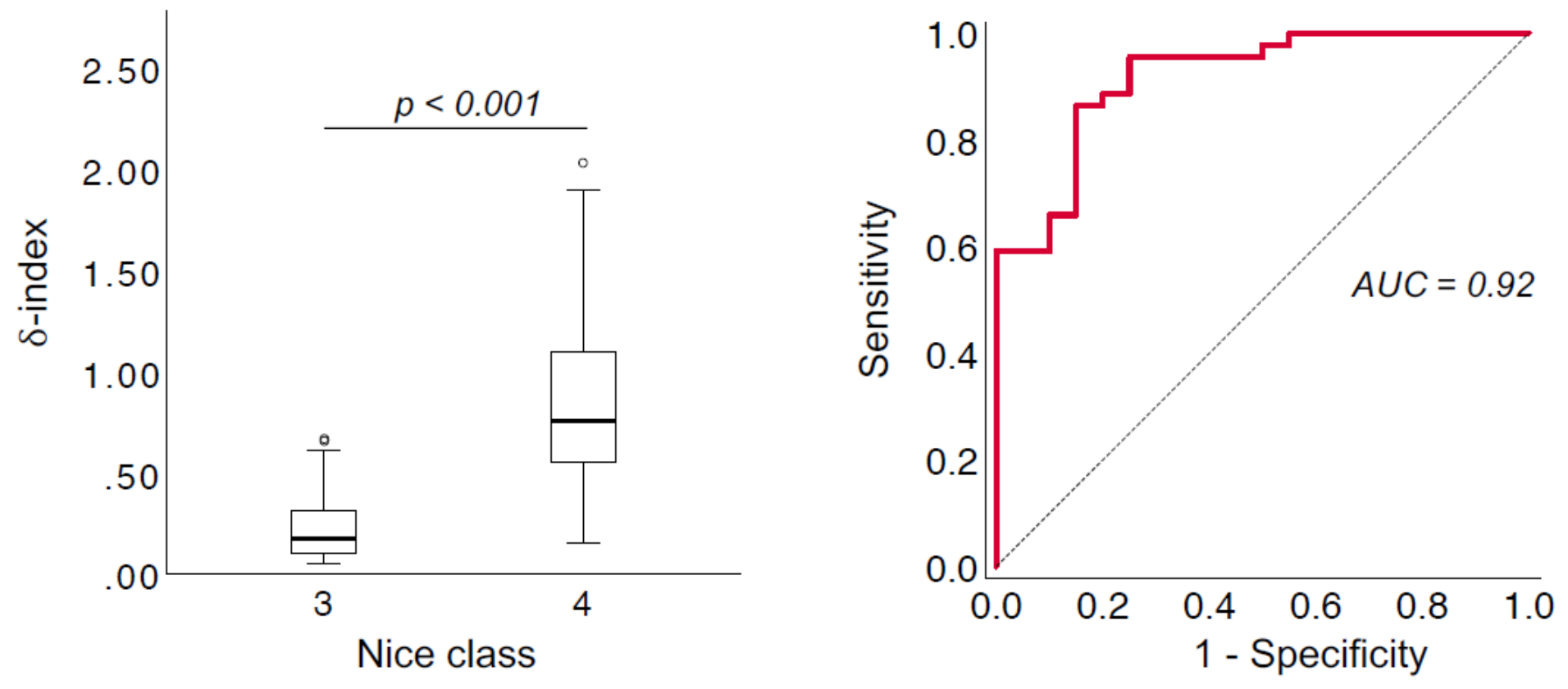

Figure 5

Differentiation of patients with PH due to lung disease and/or hypoxia and patients with CTEPH based on the $\delta$-index and diagnostic accuracy of the $\delta$-index for CTEPH based on AUC analysis. CTEPH, chronic thromboembolic pulmonary hypertension. 
Step 1: Identification of Nice class 3 and 4

IDI-based assessment of malperfused lung areas and evaluation of ID skewness in $V_{\text {PerfDef }}$
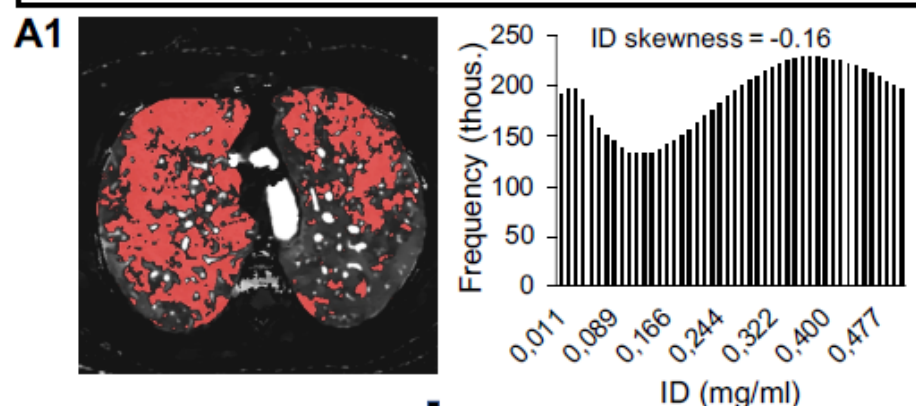

ID $(\mathrm{mg} / \mathrm{ml})$
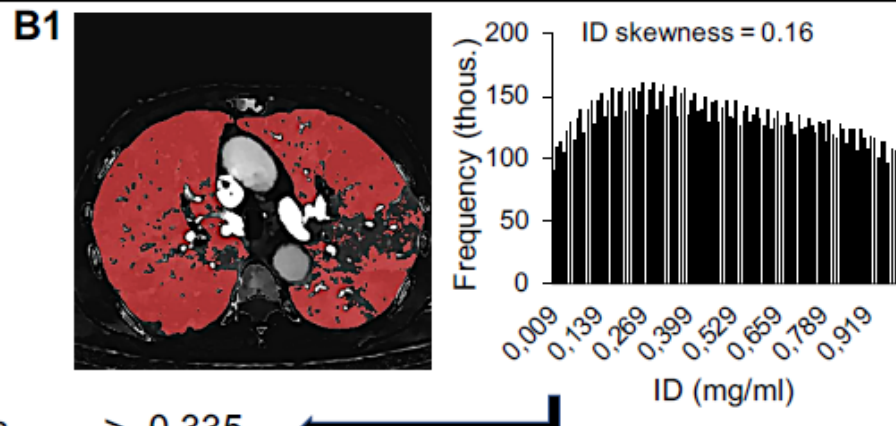

ID Skewness PerfDef $>-0.335$

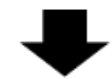

ID distribution suggestive of Nice class 3 or 4

\section{Step 2: Further subclassification}

Evaluation of corresponding pulmonary parenchyma alterations in malperfused lung areas via VNC-based lung emphysema quantification and the $\delta$-Index

A2
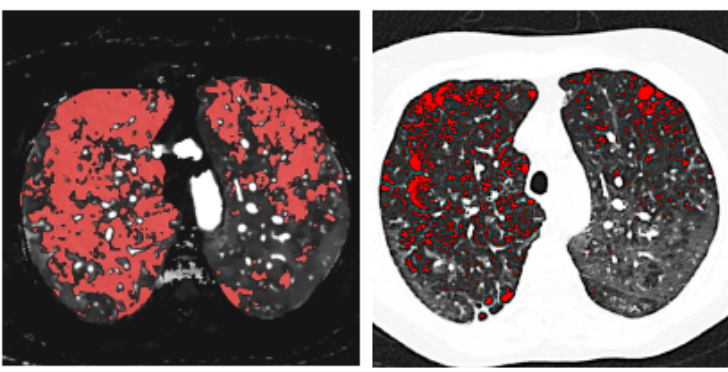

Emphysema [\% total lung volume] $=7.7$ $\delta$-Index $=0.14(<0.552)$

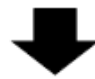

More likely diagnosis: Nice class 3
B2
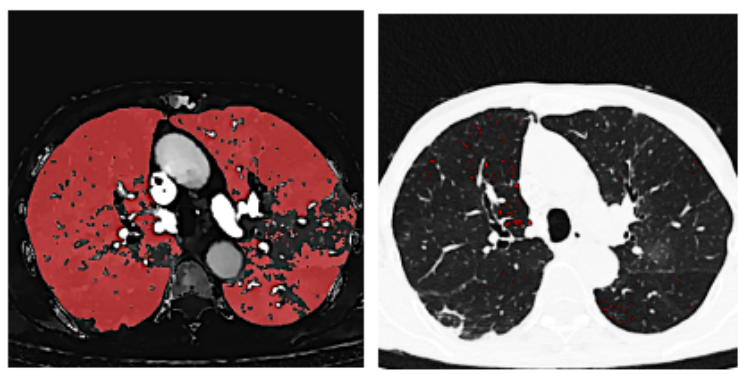

Emphysema [\% total lung volume] $=0.4$ $\delta$-Index $=1.08(>0.552)$

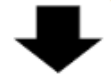

More likely diagnosis: Nice class 4

Figure 6

Stepwise approach for the identification and differentiation of Nice classes 3 (Panels A1 and A2) and 4 (Panels B1 and B2) based on ID skewness in malperfused lung areas, VNC-based quantification of emphysematous lung parenchyma and the $\delta$-index. IDI, iodine-density image; ID, iodine density; VPerfDef, malperfused lung volume; thous., thousand; (ID SkewsnessPerfDef, ID skewness in malperfused lung areas; VNC, virtual noncontrast. 